REVISTA DE LA FACULTAD

DE CIENCIAS DE LA SALUD

\title{
Médicos y depresión: una responsabilidad de todos en la sociedad
}

Doctors and depression: the responsibility of everyone in society

Médicos e depressão: uma responsabilidade de todos na sociedade

María Cristina Martínez-Ávila, MD., Esp. ${ }^{1}$ (D) Liz Adriana Castillo-Porras, MD., Esp. ${ }^{1}$ (D), Maryudi Velandia-Plata, MD., Esp. ${ }^{2}$ (D), María Gabriela RamírezGuerrero, MD., Esp. ${ }^{1}$ (D)

1. Médico, Especialista en Epidemiología, Universidad Autónoma de Bucaramanga, Floridablanca, Santander, Colombia.

2. Médico, Especialista en Oftalmología, Universidad Militar Nueva Granada, Bogotá, Cundinamarca, Colombia.

Correspondencia: María Cristina Martínez-Ávila, Médico, Especialista en Epidemiología, Universidad Autónoma de Bucaramanga. 130001.E-mail: cristina.martinezavila@gmail.com

\section{INFORMACIÓN ARTÍCULO}

DOI: https://doi.org/10.29375/01237047.3611

\section{Sra. editora:}

En la actualidad, los casos de depresión y, a su vez, el fatal desenlace del suicidio, son cada vez más frecuentes. De acuerdo con los datos reportados por la Organización Mundial de la Salud, cerca de 3,000 personas se suicidan cada día en el mundo (lo que equivale a una cada 30 segundos) y otras 60,000 intentan hacerlo, pero no lo logran, haciendo que tal situación cobre gran importancia (1). Tal vez, debido a que se esté dejando de lado, omitiendo, la problemática de salud mental es vista como un problema que nos estorba y no como un problema que requiere pronta atención y solución. De esta problemática los médicos no están exentos: se puede ver, en este grupo, un notorio aumento en tasas de depresión y ansiedad, por encima de lo observado en la población en general. Por lo tanto, el tema del suicidio demanda atención; siendo así, se enfocará su abordaje hacia el personal de salud y, entre estos, a los médicos.

La población general concibe la figura del médico como ese ser infalible, inequívoco, indefectible y seguro, a quien, probablemente, no le hace 
falta nada en su vida y que, adicionalmente, por tener conocimientos en salud, puede asumir mejor su autocuidado y que siempre debe saber qué medicarse para cualquier síntoma que aparezca en su salud. No obstante, los médicos tienen una tasa de suicidio más alta con respecto a otras profesiones. Según literatura reciente, presentada en la conferencia de la American Psychiatric Association, hasta 40 médicos por cada 10,0000 mueren por suicidio al año, lo cual corresponde a más del doble de la población general (2). Adicionalmente, en un estudio se demostró que es más frecuente la depresión en estudiantes de medicina y residentes, con un 15 a $30 \%$ de detección positiva de síntomas depresivos (3). Rotenstein et al. en un estudio tipo metanálisis realizado en el 2016, determinaron que la prevalencia general de depresión o síntomas depresivos en los estudiantes de medicina fue del 27.2 $\%$, y que la prevalencia general de ideación suicida fue del $11.1 \%$; de aquellos estudiantes en los cuales fue positiva la depresión, solo un $15.7 \%$ buscó tratamiento psiquiátrico (4). Según el conocimiento de los autores, existen pocos estudios que evalúen la frecuencia de conductas suicidas en estudiantes de pregrado de medicina de Latinoamérica y Colombia. Un estudio realizado en el 2013 en las escuelas de medicina de Bucaramanga reveló que la prevalencia de ideación suicida fue del $15.7 \%$, en tanto que el $5 \%$ tuvo por lo menos un intento franco de terminar con su vida, o sea, intento de suicidio (5). Esta situación puede estar en relación con la gran carga laboral y emocional que la profesión acarrea: jornadas con mayor intensidad horaria que el promedio de las otras profesiones. También puede ser debida a la mala remuneración, además de la poca cantidad de médicos que hay en los servicios de consulta externa, cirugía y urgencias, en donde hay sobrecupo en el número de pacientes por atender, sumado a la responsabilidad que conlleva tratar con seres humanos y el miedo a las posibles demandas que puedan aparecer. Todo esto tras haber sacrificado parte de su vida y su familia durante el pregrado y, para quienes tienen la posibilidad, el postgrado.

El agotamiento entre los profesionales de la salud se caracterizaporagotamientoemocional,despersonalización y niveles reducidos de logros personales, relacionados con la insatisfacción laboral, mala calidad de atención o intención de retirarse de la práctica médica. En un estudio publicado en Suiza se evaluaron 1,732 médicos de atención primaria, entre estos, médicos generales y especialistas. En un análisis multivariable, se encontraron niveles más altos de salud mental en los encuestados con mayor satisfacción laboral con sus ingresos y prestigio social (standardized beta coefficients SBC 1.04), relaciones profesionales (SBC 0.57) y en los encuestados con menor agotamiento emocional (SBC -4.98) y logros personales más altos (SBC 1.72). Los niveles más altos de estas dimensiones de satisfacción laboral también "amortiguaron" el impacto del agotamiento emocional en la salud mental del médico (6).

Por años, se ha descrito en la literatura una tasa mayor de suicidio entre médicos que en la población general, principalmente, en mujeres médicas. Entre los factores asociados descritos se encuentran: trastornos del estado del ánimo, abuso de drogas, relacionados con el alcohol, patrones cognitivos $(61 \%$ de los médicos cree que el suicidio es racional bajo determinadas circunstancias), factores psicosociales (conflicto de roles, hogar, deudas, exigencia laboral, estatus social) y características de la personalidad (obsesivo compulsivo, distímico, orientado al éxito, introvertido, ansioso y autosuficiencia excesiva) (7). Impulsados por estos hallazgos, en nuestro entorno laboral actual con múltiples factores estresantes en la práctica de la medicina, es importante estar atentos a nuestra salud emocional y la de los colegas, ser más sensibles a problemas mentales, estar dispuestos a pedir ayuda y ofrecer apoyo a quien lo necesite, para evitar pérdidas humanas valiosas en la medicina.

Es imprescindible que se tome conciencia de esta situación, debido a que la mayoría de los profesionales la pueden llegar a sufrir en silencio, con repercusiones familiares, sociales, emocionales y laborales. Por esto, se sugiere la creación de estrategias de prevención, detección temprana en cuanto depresión, cambios de ánimo y signos de alarma sugestivos de vulnerabilidad para que dichos profesionales reciban atención oportuna que evite llegar al suicidio, al síndrome de burnout o a desenlaces indeseados. Adicionalmente, se hace evidente la necesidad de programas dirigidos a la promoción de la salud mental y la prevención de enfermedades mentales que incluyan control emocional, manejo del estrés, técnicas de relajación en situaciones de mayor presión, no solo para contener los momentos o períodos de vulnerabilidad, incremento de demanda asistencial, ansiedad y estrés laboral. sino también para preservar la salud del médico y de los profesionales sanitarios y, de este modo, mejorar la calidad asistencial.

\section{Referencias}

1. World Health Organization. Suicidio [Internet]. World Health Organization; 2018 [citado 18 de mayo de 2019]. Recuperado a partir de: https://www.who.int/ es/news-room/fact-sheets/detail/suicide

2. Vogel L. Has suicide become an occupational hazard of practising medicine?. CMAJ [Internet]. 2018 [cita- 
3. Mingote JC, Crespo D, Hernández M, Navío M, Rodrigo C. Suicide prevention in doctors. Med Segur Trab [Internet]. 2013 [citado 5 de abril de 2019];59(231):176-204. Recuperado a partir de: http:// scielo.isciii.es/pdf/mesetra/v59n231/especial.pdf

4. Rotenstein LS, Ramos MA, Torre M, Segal JB, Peluso MJ, Guille C, et al. Prevalence of depression, depressive symptoms, and suicidal ideation among medical students: a systematic review and meta-analysis. JAMA [Internet]. 2016 [citado 23 de mayo de 2019];316(21):2214-36. doi: 10.1001/ jama.2016.17324

5. Pinzón-Amado A, Guerrero S, Moreno K, Landínez C, Pinzón J. Ideación suicida en estudiantes de medicina: prevalencia y factores asociados. Rev Colomb.Psiquiatr [Internet]. 2013 [citado 5 de marzo de 2019];42(Suppl 1):47-55. Recuperado a partir de: http://www.scielo.org.co/scielo.php?script=sci_arttext\&pid=S0034-74502013000500007\&lng=en.
6. Bovier PA, Arigoni F, Schneider M, Gallacchi MB. Relationships between work satisfaction, emotional exhaustion and mental health among Swiss primary care physicians. Eur J Public Health [Internet]. 2009 [citado 6 de junio de 2019]. doi: 10.1093/eurpub/ ckp056

7. Sansone RA, Sansone LA. Physician suicide: a fleeting moment of despair. Psychiatry [Internet]. 2009 [citado 3 de marzo de 2019];6(1):18-22 Recuperado a partir de: https://www.ncbi.nlm.nih.gov/pubmed/19724738 